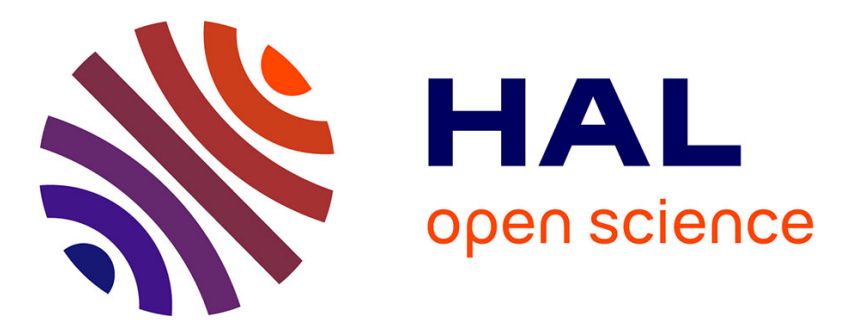

\title{
Mechanisms of non-modal energy amplification in channel flow between compliant walls
} Jérôme Hoepffner, Alessandro Bottaro, Julien Favier

\section{To cite this version:}

Jérôme Hoepffner, Alessandro Bottaro, Julien Favier. Mechanisms of non-modal energy amplification in channel flow between compliant walls. Journal of Fluid Mechanics, 2010, 642, pp.489-507. 10.1017/S0022112009991935 . hal-01073478

\section{HAL Id: hal-01073478 https://hal.science/hal-01073478}

Submitted on 9 Oct 2014

HAL is a multi-disciplinary open access archive for the deposit and dissemination of scientific research documents, whether they are published or not. The documents may come from teaching and research institutions in France or abroad, or from public or private research centers.
L'archive ouverte pluridisciplinaire HAL, est destinée au dépôt et à la diffusion de documents scientifiques de niveau recherche, publiés ou non, émanant des établissements d'enseignement et de recherche français ou étrangers, des laboratoires publics ou privés. 


\section{Mechanisms of non-modal energy amplification in channel flow between compliant walls}

\section{JÉRÔME HEPFFNER ${ }^{1}$, ALESSANDRO BOTTARO ${ }^{2}$ A ND JULIEN FAVIER}

${ }^{1}$ Dept of Mechanical Engineering, Keio University, 3-14-1 Hiyoshi, Yokohama, 223-8522, Japan. (Present affiliation: Institut Jean le Rond D'Alembert, UMR 7190, Université Pierre et Marie Curie, Paris, France. email: jerome.hoepffner@upmc.fr)

${ }^{2}$ DICAT, Università di Genova, Via Montallegro 1, 16145, Genova, Italy.

(Received May 28, 2008)

The mechanisms leading to large transient growth of disturbances for the flow in a channel with compliant walls are investigated. The walls are modeled as thin spring-backed plates, and the flow dynamics is modeled using the Navier-Stokes equations linearised round the Poiseuille profile. Analysis for streamwise-invariant perturbations show that this fluidstructure system can sustain oscillatory energy evolution of large amplitude, in the form of spanwise standing waves. Such waves are related to the travelling waves which a free wall can support, modified to account for an 'added mass' effect. Simple scaling arguments are found to provide results in excellent agreement with computations of optimal disturbances, for low to moderate values of the stiffness parameter characterising the compliant surface.

\section{Introduction}

The original observations by Gray (1936) of swimming dolphins, and the inference that their high propulsive efficiency is - at least partially - due to the compliance of their skin, has attracted much attention on the effect of flexible boundaries on transitional and turbulent flows. Studies on the subject started with Kramer $(1957,1960)$ who conducted pioneering tests by towing a torpedo-like model, equipped with an artificial skin modeled on the structure of the dolphin's dermis and epidermis, with a motor boat in Long Beach harbor, California. Kramer's experiments could not be successfully replicated in the years to follow (giving rise to the so-called Kramer controversy), reflecting the extreme sensitivity of the problem to the properties of the compliant wall and the environmental conditions; however, the appealing idea of tailoring wall characteristics to control the fluid flowing over it did not die out, and successive waves of funding resources in Russia, the United States, some European countries and Japan were geared towards studies of optimal compliant coatings for drag reduction over aerial and marine vehicles (cf. Chapt. 7 of Gad-el Hak (2000), for an excellent overview of the subject).

From the theoretical/computational point of view, the problem is a classical fluidstructure interaction problem, with potential difficulties related to the coupling between the solver dedicated to the solid part (where the Navier equations must be used) and that used in the fluid domain (where the Navier-Stokes equations apply). An alternative is represented by the use of thin plate/shell theory to model the behaviour of the solid. When the deformation and the displacement of the bounding plate are sufficiently small, a 
simple linearized problem can be derived by replacing the no-slip condition on the plate's actual boundary with equivalent boundary conditions applied over a fictitious wall. The study of the growth/decay of small disturbances developing in the flow past compliant surfaces (modeled as thin plates) has been given a strong impulse by Carpenter \& Garrad (1986) and Carpenter \& Garrad (1985), after the initial theories laid out by Benjamin (1960) and Landahl (1962). Carpenter and Garrad focussed on the stability of boundary layer flows over Kramer-type compliant walls. The motivation for their work was the so-called Gray's paradox which holds that the sustained swimming speed of some species of dolphin can only be maintained by the extraordinary laminar flow properties of their skins (see e.g. Carpenter et al. (2000)). Results demonstrated that a suitable choice of material properties could delay transition induced by viscous instabilities, but conflicting requirements on the properties of the wall coating were needed to yield appreciable positive effects on the flow-induced surface instabilities. In practical applications, the presence of wall-based instabilities (which can take many different forms) would limit the potential for obtaining a transition delay. Today, it is believed that Gray's premises were flawed (Fish \& Lauder (2006)). Drag on the dolphin is not kept low by the maintenance of a laminar boundary layer; evidence indicates that streamlining of the body causes the turbulent boundary layer over the dolphin's skin to remain fully attached (with a consequent drag benefit against the separated-flow configuration). According to Fish \& Lauder (2006), drag reduction is also associated to so-called behavioural mechanisms of the dolphin, related mainly to its breathing habits. For a comprehensive review of compliant wall instabilities, the reader is referred to the book edited by Carpenter \& Pedley (2003).

In these studies, the analysis of the possibility of transition to turbulence was assessed through the search of unstable eigenmodes of the fluid/walls system. It is known on the other hand that transition to turbulence can occur in flows that do not have any unstable eigenmode. Such subcritical transition scenario is linked to transient mechanisms of energy transfer from the base flow to perturbations. This idea was successful for channel flow and boundary layers, where initial conditions consisting in elongated streamwise vortices were found to interact with the base flow to generate strong streaks of streamwise vorticity. This is known as the lift-up mechanism, see Schmid \& Henningson (2001).

We briefly illustrate this effect in a Poiseuille flow between two rigid plates. The base flow is a parabola $U(y)=1-y^{2}$, and we assume perturbations of small amplitude on the streamwise $u$, cross-shear $v$ and spanwise $w$ velocity components, and pressure $p$. The evolution in time of these small perturbations is described by the Navier-Stokes equations linearized about the base flow profile

$$
\begin{aligned}
& u_{t}+U u_{x}+U_{y} v=-p_{x}+\Delta u / R e \\
& v_{t}+U v_{x}=-p_{y}+\Delta v / R e \\
& w_{t}+U w_{x}=-p_{z}+\Delta w / R e \\
& u_{x}+v_{y}+w_{z}=0 .
\end{aligned}
$$

Subscripts $t, x, y, z$ denote partial derivatives in these directions, and $\Delta=\partial_{x x}+\partial_{y y}+\partial_{z z}$ is the Laplacian operator.

In the lift-up mechanism, energy is transferred from the base flow to the perturbations through the effect of the cross-shear velocity $v$ : the base flow is advected up or down. This effect is best seen for perturbations that are uniform in the streamwise direction $x$, 
and for a fluid of negligible viscosity. Then (1.1) takes the simpler expression

$$
\begin{aligned}
u_{t}+U_{y} v & =0, \\
v_{t} & =-p_{y}, \\
w_{t} & =-p_{z}, \\
v_{y}+w_{z} & =0 .
\end{aligned}
$$

The system is now decoupled: the flow $(v, w)$ in a plane normal to the streamwise direction is subject to no-penetration at the walls and incompressibility, and is ignorant of the base flow $U$. On the other hand, the cross-shear velocity $v$ can create streamwise velocity perturbation by advection of the base flow $U_{y} v$. With the assumption of small amplitude perturbations, the base flow acts as an infinite source of $u$, provided $v$ is there to extract it through $(1.2 a)$. We can now ask the question: what is a good initial condition for this lift-up effect to be fully effective? It turns out that the best condition is when the initial field consists of time-independent streamwise vortices, which yield $u$ growing linearly in time as

$$
u=-t U_{y} v
$$

for a vanishing initial value of $u$. The effect of fluid viscosity will then be as follows: a streamwise vortex shall dissipate slowly, its lifetime scaling as the Reynolds number. For a period of time of order $R e, u$ grows linearly and thus reaches an amplitude of order $R e$ (an energy of order $R e^{2}$ ). When the initial vortex dies out, the source of $u$ dies too, and $u$ dissipates because of viscosity: this mechanism is transient. If - on the other hand the amplitude reached is large enough, transition to turbulence can happen. With active viscous dissipation, the most long-lived streamwise vortices should have an aspect ratio of about one: not thinner or wider than tall. In $y$ the vortices are confined betwen the walls (at $y= \pm 1$ ), thus their span should be of about 2 units of length.

Let us now turn to the case of our interest: a channel flow with flexible walls. Here too, the only source of energy is the base flow; here too, energy can be extracted only through cross-shear advection by $v$. What is new now, is that the walls can move: there can be cross-shear velocity next to the walls, precisely where the shear is largest. Indeed, with $U=1-y^{2}$, we have $U_{y}=-2 y$. We can imagine long lived $v, w$ motion which are not streamwise vortices. In a sense, the no-penetration condition at $y= \pm 1$ is now alleviated, and this was the most constraining condition in the rigid wall channel. On the other hand, now the dynamics of the $v, w$ perturbations is slaved to the dynamics of the flexible walls.

The object of the present paper is to analyse whether the wall dynamics and its coupling with the flow can allow for energy transfer from the base flow, and to characterize this transfer. We shall see that wall spanwise standing waves-built from two oppositely propagating traveling waves - will play an important role.

\section{System description}

Following Carpenter \& Garrad (1986), the Kramer-type wall is modeled as a springbacked flexible plate. Its deviation $\eta$ from rest at $y= \pm 1$ obeys the equation:

$$
m \eta_{t t}+\frac{d}{R e} \eta_{t}+\frac{B \triangle^{2}-T \triangle+K}{R e^{2}} \eta= \pm\left. p\right|_{\text {wall }},
$$

with $\triangle=\partial_{x x}+\partial_{z z}$ the 2D Laplacian operator, and the Reynolds number $R e$ defined on channel half-height, centreline velocity and dynamic viscosity. The fluid acts on the plate through the pressure: $p$ forces this equation with a positive sign at the top wall 
and a negative sign at the bottom wall (high pressure pushes the top wall up and the bottom wall down). The other parameters are the wall density $m$, the wall damping coefficient $d$, the wall flexural rigidity $B$, the wall tension $T$ and the spring stiffness $K$ (Davies \& Carpenter 1997). A similar model, with however the inclusion of the normal viscous stresses in the forcing term on the right-hand-side of (2.1), has been considered, for example, by Wiplier \& Ehrenstein (2001). The addition of the normal viscous stress forcing will slightly change the expression of the total system energy in $\S 2.2$, but we found that the energy exchanges between the fluid and the walls described by these terms do not play a significant role in the transient mechanisms described in the present paper.

For this wall model, there are five parameters in addition to the Reynolds number. Since this is a large parameter space, we fix $B=4 K, T=0$ and $m=2$, following Davies \& Carpenter (1997). With this choice of $m$, the mass of the walls is comparable to that of the fluid (which has unit density), so that inertia of both media play a role in the system's dynamics. When considering the evolution of wave packets, the interplay of tension, flexibility and spring stiffness, $T, B$ and $K$, influences the group velocity. For the present investigation on the other hand, we consider simple harmonic waves, and thus in (2.1) it is mainly the balance between the coefficients of the acceleration term $\eta_{t t}$ and position, $\eta$, that affect the behaviour of wall waves. For this reason, we change the wall properties mainly by varying the spring stiffness $K$. We have nonetheless performed additional computations with others parameters to assess the wider relevance of our conclusions. In (2.1), the wall equation was made dimensionless on the basis of characteristic flow quantities (which explains the presence of the factors $1 / R e$ and $1 / R e^{2}$ ), in order to maintain constant properties of the wall while changing the Reynolds number of the flow.

\subsection{Eigenmodes}

The flow dynamics is described by the Navier-Stokes equations linearized about the Poiseuille profile. Assuming small wall displacement from rest, the no-slip boundary conditions on $u, v, w$ at the displaced wall can be enforced by means of inhomogeneous boundary conditions at $y= \pm 1$ through a Taylor expansion. If we denote $u^{\prime}=u+U, v^{\prime}=$ $v, w^{\prime}=w$ the fluid velocity (base flow plus perturbation), we have, for instance for the top wall:

$$
\begin{array}{lll}
u^{\prime}(1+\eta)= & 0=u^{\prime}(1)+\eta u_{y}^{\prime}(1)+\mathcal{O}\left(\eta^{2}\right) & \approx u(1)+\eta U_{y}(1) \\
v^{\prime}(1+\eta)= & \eta_{t}=v^{\prime}(1)+\eta v_{y}^{\prime}(1)+\mathcal{O}\left(\eta^{2}\right) & \approx v(1) \\
w^{\prime}(1+\eta)=0 & =w^{\prime}(1)+\eta w_{y}^{\prime}(1)+\mathcal{O}\left(\eta^{2}\right) & \approx w(1)
\end{array}
$$

where we have used $U(1)=0$ and neglected second order terms. This leads to the boundary conditions

$$
\left.u\right|_{\text {wall }}+\left.\eta U_{y}\right|_{\text {wall }}=0,\left.\quad v\right|_{\text {wall }}=\eta_{t},\left.\quad w\right|_{\text {wall }}=0 .
$$

To analyse the dynamics of this system, we can consider individual wavenumber pairs in the streamwise and spanwise directions, based on the normal mode decomposition

$$
f(x, y, z, t)=\frac{1}{2}\left(\hat{f}(y) \mathrm{e}^{i(\alpha x+\beta z-\omega t)}+\text { c.c. }\right)
$$

for $u, v, w, p$, and $\eta$, where c.c. denotes complex conjugates. Introducing this decomposition into the dynamic equations, we obtain a generalized eigenmode problem. First for the fluid

$$
-\mathrm{i} \omega E \hat{q}=A \hat{q}
$$


with

$$
\hat{q}=\left(\begin{array}{c}
\hat{u} \\
\hat{v} \\
\hat{w} \\
\hat{p}
\end{array}\right), \quad E=\left(\begin{array}{cccc}
1 & 0 & 0 & 0 \\
0 & 1 & 0 & 0 \\
0 & 0 & 1 & 0 \\
0 & 0 & 0 & 0
\end{array}\right), \quad A=\left(\begin{array}{cccc}
S & -U_{y} & 0 & -i \alpha \\
0 & S & 0 & -\partial_{y} \\
0 & 0 & S & -\mathrm{i} \beta \\
\mathrm{i} \alpha & \partial_{y} & \mathrm{i} \beta & 0
\end{array}\right)
$$

where $S=-\mathrm{i} \alpha U+\left(\partial_{y y}-k^{2}\right) / R e$ is a convection/diffusion operator, and $k^{2}=\alpha^{2}+\beta^{2}$. For the walls, we first transform (2.1) into a differential system, introducing $\gamma=\eta_{t}$ :

$$
\mathrm{i} \omega\left(\begin{array}{cc}
m & 0 \\
0 & 1
\end{array}\right)\left(\begin{array}{c}
\hat{\gamma} \\
\hat{\eta}
\end{array}\right)=\left(\begin{array}{cc}
-d / R e & -\left(B k^{4}+T k^{2}+K\right) / R e^{2} \\
1 & 0
\end{array}\right)\left(\begin{array}{c}
\hat{\gamma} \\
\hat{\eta}
\end{array}\right)+\left(\begin{array}{c} 
\pm\left.\hat{p}\right|_{\text {wall }} \\
0
\end{array}\right)
$$

The systems (2.5) and (2.6) for both walls are then coupled through the boundary conditions and the wall pressure forcing. The state of our coupled system is now the vector

$$
\left(\hat{u}, \hat{v}, \hat{w}, \hat{p}, \hat{\gamma}_{t o p}, \hat{\eta}_{t o p}, \hat{\gamma}_{b o t}, \hat{\eta}_{b o t}\right)^{T}
$$

where the subscripts $\cdot_{\text {top }}$ and ${ }^{b o t}$ identify the variables relative to the top and bottom channel walls. The velocity components and pressure $\hat{u}, \hat{v}, \hat{w}, \hat{p}$ are discretized in the $y$ direction using Chebychev collocation, see Weideman \& Reddy (2000), and the numerical

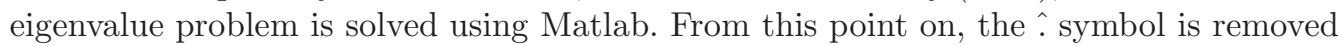
without ambiguity.

\subsection{Energy budget for the fluid/walls system}

We now consider the system's energy, where by system we denote the flow plus the walls. The total energy is composed by the flow kinetic energy, plus the wall kinetic and potential energies. We use the Reynolds-Orr equation, modified to account for the inhomogeneous boundary conditions, to derive a relation for the energy evolution in time (see e.g. Guaus \& Bottaro 2007):

$$
\begin{aligned}
& \overbrace{\left(\frac{1}{2} \int_{y} \overline{u^{2}+v^{2}+w^{2}} \mathrm{~d} y\right)_{t}}^{\left(E_{K}^{f}\right)_{t}}+[\overline{v p}]_{\mathrm{bot}}^{\text {top }}= \\
& -\underbrace{\int_{y} U_{y} \overline{u v} \mathrm{~d} y}_{\text {Reynolds stress term }}+\underbrace{\frac{1}{R e}\left[\overline{u u_{y}+v v_{y}}\right]_{\text {bot }}^{\text {top }}}_{\begin{array}{c}
\text { Extra-energy removal } \\
\text { and supply term }
\end{array}}-\underbrace{\frac{1}{R e} \int_{y} \overline{\boldsymbol{\omega} \cdot \boldsymbol{\omega}} \mathrm{d} y}_{\begin{array}{c}
\text { Fluid viscous } \\
\text { damping term }
\end{array}},
\end{aligned}
$$

where the overline denotes averaging in the horizontal plane, the square brackets indicate the difference between the top and bottom wall values and $\boldsymbol{\omega}$ is the flow vorticity. The term labeled $E_{K}^{f}$ is the usual flow kinetic energy density. The Reynolds stress term can generate or withdraw energy from the perturbation due to exchange of energy with the base flow. The viscous damping term always subtracts energy, and there is an additional term that can give or take energy away through interactions of the fluid with the walls.

The term $[\overline{v p}]_{\text {bot }}^{\text {top }}$ corresponds to energy exchanges between the flow perturbations and the wall through the work of pressure forces. We can use this term to isolate the expressions of the wall kinetic and potential energy. We substitute the value of $v$ at the wall by the time derivative of the wall position from the boundary conditions (2.3), and 
$p$ by the wall dynamic equation (2.1). We thus have:

$$
\begin{aligned}
{[\overline{v p}]_{\text {bot }}^{\text {top }} } & =\sum_{\text {bot }}^{\text {top }} \overline{\eta_{t}\left(m \eta_{t t}+\frac{d}{R e} \eta_{t}+\frac{B \triangle^{2}-T \triangle+K}{R e^{2}} \eta\right)} \\
& =\sum_{\text {bot }}^{\text {top }}\{\underbrace{m\left(\frac{\overline{\eta_{t}^{2}}}{2}\right)_{t}}_{\left(E_{K}^{w}\right)_{t}}+\underbrace{\frac{d}{R e} \overline{\eta_{t}^{2}}}_{\begin{array}{l}
\text { Viscous damping } \\
\text { term in the wall }
\end{array}}+\underbrace{\left(\frac{B \overline{(\triangle \eta)^{2}}+T \overline{\nabla \eta \cdot \nabla \eta}+K \overline{\eta^{2}}}{2 R e^{2}}\right)}_{\left(E_{P}^{w}\right)_{t}}\},
\end{aligned}
$$

with $\nabla=\left(\partial_{x}, \partial_{z}\right)^{T}$ is the 2D gradient operator. In this expression, $E_{K}^{w}$ and $E_{P}^{w}$ are the wall kinetic and potential energies. We obtained the expression of the flexion and tension contribution to the wall potential energy using integration by parts in $x$ and $z$, using the assumed spatial periodicity of $\eta$

$$
\overline{\eta_{t}\left(\triangle^{2} \eta\right)}=\frac{1}{2} \overline{\left((\triangle \eta)^{2}\right)_{t}}, \quad \overline{\eta_{t}(\triangle \eta)}=-\frac{1}{2} \overline{(\nabla \eta \cdot \nabla \eta)_{t}} .
$$

The total perturbation energy of the flow/walls system is thus:

$$
\bar{E} \triangleq \underbrace{\frac{1}{2} \int_{y} \overline{u^{2}+v^{2}+w^{2}} \mathrm{~d} y}_{\text {Flow }}+\underbrace{\sum_{\text {bot }} \frac{1}{2}\left(m \overline{\eta_{t}^{2}}+\frac{B \overline{(\triangle \eta)^{2}}+T \overline{\nabla \eta \cdot \nabla \eta}+K \overline{\eta^{2}}}{R e^{2}}\right)}_{\text {Walls }} .
$$

This expression of the energy will be useful in the sequel, since this is the term for which optimization will be sought. The total energy budget is then from (2.7):

$$
\overline{E_{t}}=\underbrace{-\int_{y} U_{y} \overline{u v} \mathrm{~d} y+\frac{1}{R e}\left[\overline{u u_{y}+v v_{y}}\right]_{\mathrm{bot}}^{\mathrm{top}}}_{\begin{array}{c}
\text { Energy exchange with base flow } \\
\text { and through the walls }
\end{array}} \underbrace{-\frac{1}{R e} \int_{y} \overline{\boldsymbol{\omega} \cdot \boldsymbol{\omega}} \mathrm{d} y-\sum_{\text {bot }}^{\text {top }} \frac{d}{R e} \overline{\eta_{t}^{2}}}_{\begin{array}{c}
\text { Viscous damping within the fluid } \\
\text { and the walls }
\end{array}} .
$$

Note that this expression of the energy budget depends on the fact that we neglect the normal stress in the wall forcing in (2.1). For a single Fourier mode in the horizontal plane, with wavenumber $(\alpha, \beta)$, the expression of the total energy becomes

$$
\hat{E}_{k}=\frac{1}{4} \int_{y}\left(\hat{u} \hat{u}^{\star}+\hat{v} \hat{v}^{\star}+\hat{w} \hat{w}^{\star}\right) \mathrm{d} y+\sum_{\text {bot }}^{\text {top }} \frac{1}{4}\left(m \hat{\eta}_{t} \hat{\eta}_{t}^{\star}+\frac{B k^{4}+T k^{2}+K}{R e^{2}} \hat{\eta} \hat{\eta}^{\star}\right),
$$

where $\hat{u}, \hat{v}, \hat{w}$ and $\hat{\eta}$ are the eigenfunction amplitudes (function of $y$ only), and $\star$ denotes complex conjugate.

The implication of the chosen expression of the metrics to be optimised for is as follows: if the wall contribution had not been included in the definition of the energy, an initial condition with little flow perturbation but large wall deformation might be able to induce a large growth of energy, as energy stored in the wall would be released through the boundaries. We consider it more meaningful to use $E$ as functional for the optimisation. The only possible source of energy for the perturbations is then the base flow. For the perturbations, energy can be transferred during the time evolution between the flow and the flexible plates, mostly through the work of the pressure at the walls. 


\section{Wall waves}

The mechanisms that are discussed in the following sections are related to wall oscillations. A flexible plate taken by itself can sustain oscillations in the form of travelling waves. These waves are described by a dispersion relation, derived from the plate's dynamic equation. If two such plates are now used to confine fluid in a channel, the travelling waves inherent to the plate's dynamic will persist despite the presence of the fluid. An associated travelling wave will be observed in the fluid, induced by the plate's motion. We will see in the present section that this coupled motion will become an eigenmode of the fluid/walls system. Clearly, the exact properties of the wave will be affected by the presence of the fluid, mainly in the form of an added mass effect. The flow profile being dependent on the wall wavelength, this added mass effect will vary. In addition, we will be able to distinguish two different symmetries: sinuous, when the walls move antisymetrically with respect to the centreline, and varicose when the walls' motion is symmetrical.

We are particularly interested in the behaviour of perturbations which are infinitely elongated in the streamwise direction $x$ (streamwise wavenumber $\alpha=0$ ). In this case, in fact, equation (1.1) becomes

$$
\begin{array}{lrr}
u_{t}+U_{y} v & = & \Delta u / R e \\
v_{t} & = & -p_{y}+\Delta v / R e \\
w_{t} & = & -p_{z}+\Delta w / R e \\
v_{y}+w_{z} & = & 0 .
\end{array}
$$

As previously noted, the evolution of $v$ and $w$ - that is the flow in a spanwise plane - is decoupled from $u$, and independent of the base flow $U$. This is particularly interesting, because it means that $v$ and $w$ behave as they would in a channel without pressure gradient. In the present section we will thus consider the behaviour of waves traveling in the spanwise direction. Of course, $v$ and $w$ act on $u$, as emphasized when introducing the lift-up effect. Once the behaviour of spanwise waves is fully analysed, we will turn to this effect in $\S 5$, to better understand the results of the optimal growth computations.

Selected eigenmodes are depicted in figure 1 for the sinuous symmetry and figure 2 for the varicose symmetry. These flow fields correspond to waves travelling from right to left. For a sinuous wave of long wavelength, the motion of flow particles is mainly vertical, moving as a bulk with the walls (figure 1, top left). For short sinuous waves on the other hand, the flow motion occurs mostly in thin layers along the walls. We observe in figure 1 that these layers have an exponential profile with coefficient $-\beta$. This profile can be derived on the basis of inviscid arguments.

For the varicose mode at long wavelengths, the mechanism is different. Due to symmetry, flow particles cannot move from one wall to the other, but have to move along the channel from constricted to expanded sections. The spanwise velocity tends locally to a parabolic profile with the increase of the wavelength, while the vertical component remains relatively small. For short wavelengths, similarly to the sinuous case, a fluid layer of exponentially varying thickness forms near each wall.

From this description of the wave profiles, we can now determine the added mass effect. First, using the wall dynamical equation (2.1) without fluid (free wall), we can derive the dispersion relation, relating spatial and temporal properties of the oscillatory motion. We assume a normal mode structure for $\eta$, i.e. $\eta=\exp [i(\beta z+\omega t)]$, where $\omega$ is the angular frequency. Assuming vanishing wall damping $(d=0)$ we find

$$
m \omega^{2}=\frac{B \beta^{4}+T \beta^{2}+K}{R e^{2}} .
$$



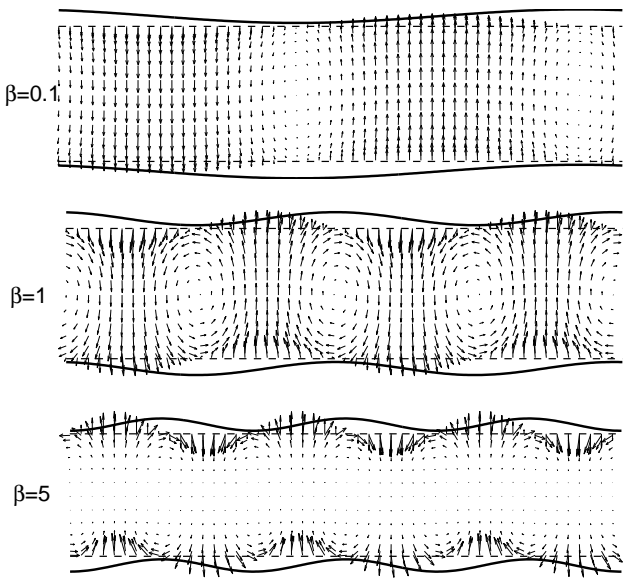

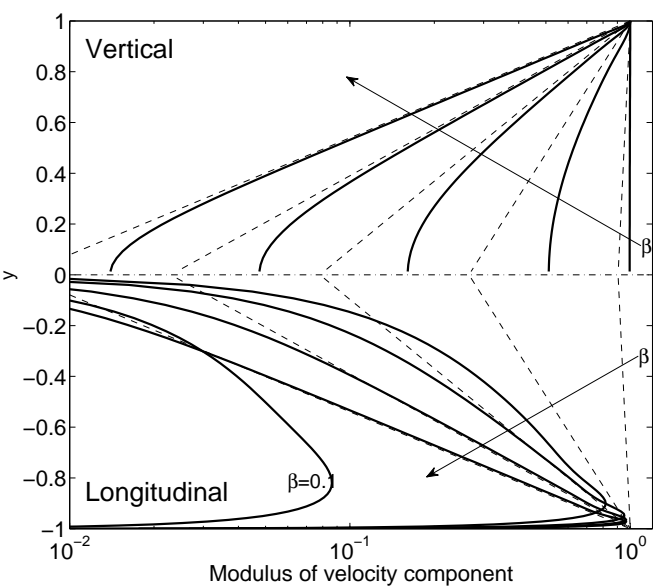

Modulus of velocity component

Figure 1. Motion of sinuous modes. Left, disturbance velocity fields for long and short wavelengths (note that the spanwise and wall normal axes are not to scale, in order to emphasize the change of the eigenmode field as the wavelength is decreased). The waves travel from right to left. Right, vertical and longitudinal velocity components (thick solid lines), compared to exponential profiles with exponential rate $-\beta$ (thin dashed lines), $\beta$ varying linearly from 0.1 to 5 , with $K=10^{5}, R e=5000$.

The flexible walls in this study have density $m=2$, and the fluid density is unity. For sinuous waves at long wavelength, fluid particle move up and down, following the wall motion. The added mass must then be unity. For short wavelengths the displaced fluid mass on each wall can be estimated from the exponential profile. Averaged over a wavelength, it should scale as:

$$
\int_{0}^{1} e^{\beta(y-1)} \mathrm{d} y=\left(1-e^{-\beta}\right) / \beta
$$

which indeed tends to unity as $\beta \rightarrow 0$. For the varicose symmetry, the dependency is the same for short wavelengths. For long waves, the amplitude of the longitudinal velocity from constricted to expanded regions is empirically found to scale as $1 / \beta^{2}$. Based on these considerations, we deduce the added masses applicable to sinuous and varicose modes that produce the expected effects both in the limits of small and large $\beta$, viz.

$$
\text { Sinuous: } m_{a}^{s}=\left(1-e^{-\beta}\right) / \beta, \quad \text { Varicose: } m_{a}^{v}=\left(1-e^{-\beta}\right) / \beta+1 / \beta^{2} \text {. }
$$

The frequency $\omega$ obtained from (3.2) is displayed in figure 3 (right frame) for free wall waves (no fluid); in the presence of fluid, the frequency of both sinuous and varicose modes, using the added mass model just outlined is also displayed. Finally, the results are compared to the computed eigenmodes, both in the presence and absence of viscosity, to ascertain the appropriateness of the model formulated. The quantitative agreement is satisfactory. The interesting fact from this analysis is that waves of the coupled fluid/walls system shall be slower than the simple wall waves. This slow down of the travelling waves will be different for the two symetries: the varicose waves at long wavelength will be very slow, due to the large added mass term $1 / \beta^{2}$.

The system's spectrum can now be characterised. The flow eigenmodes are marginally affected when compared to the rigid channel case (cf. figure 3, left frame). In addition to these modes, there are four eigenmodes that originate from the free wall traveling waves, with the eigenfrequency reduced because of an added mass effect. These are two pairs of counter-propagating waves in the channel: one pair of sinuous modes and one pair of 

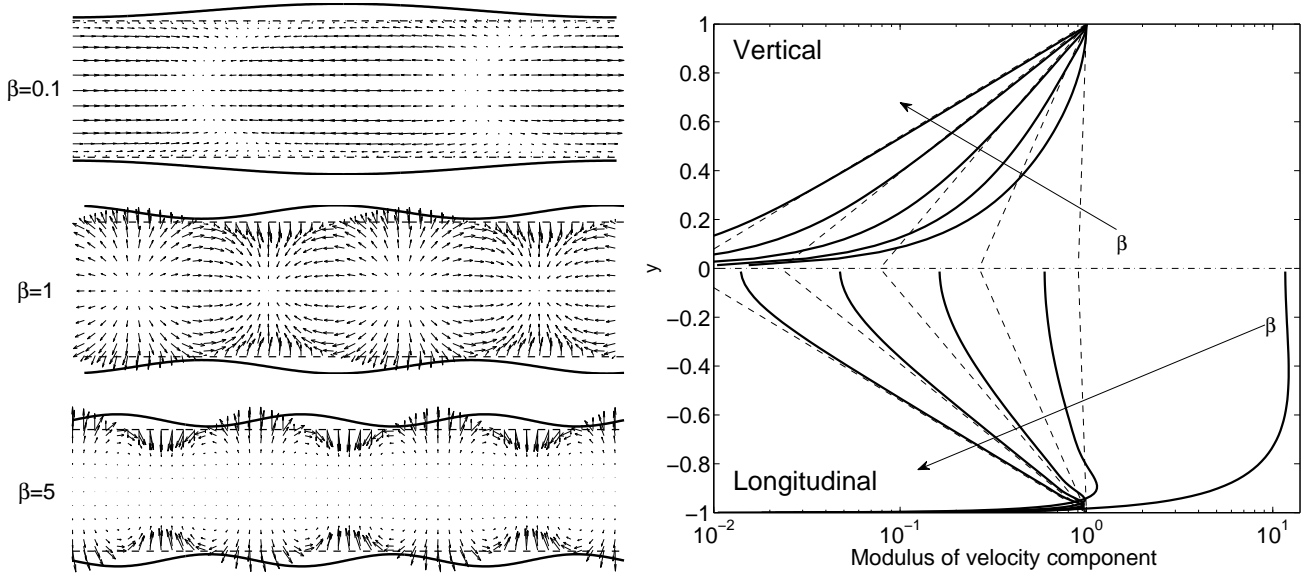

Figure 2. Motion of varicose modes. Left, disturbance velocity field for long and short wavelengths (note that the spanwise and wall normal axes are not to scale, in order to emphasize the change of the eigenmode field as the wavelength is decreased). The waves travel from right to left. Right, vertical and longitudinal velocity components (thick solid lines), compared to exponential profiles with exponential rate $-\beta$ (thin dashed lines). The parameters are those of figure 1.
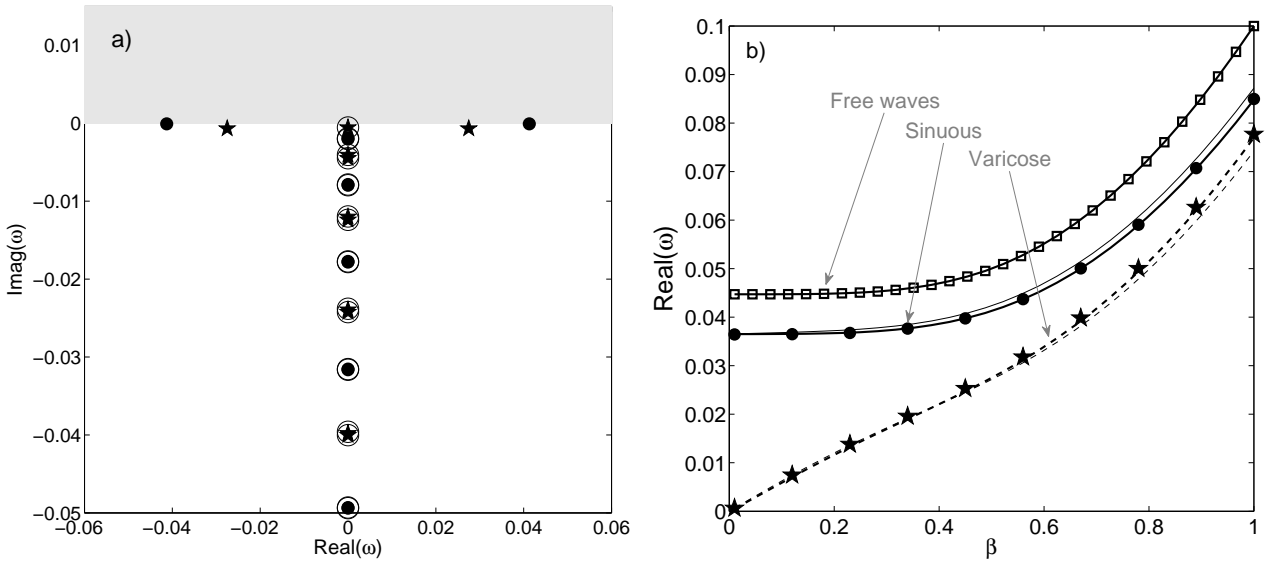

FiguRE 3. a) Spectrum at $\beta=0.5$, for the sinuous (dots) and varicose (stars) symmetries, compared to the spectrum of the rigid system (open circles). The unstable half-plane $(\operatorname{Imag}(\omega)>0)$ is shaded. b) Circular frequency $\omega$ of free wall waves (squares), eigenmodes for sinuous (thick solid) and varicose (thick dashed) waves, compared to the results obtained using the added mass model (thin lines). The eigenmodes computed with the inviscid model are drawn with circles for the sinuous and with stars for the varicose symmetry (same parameters as in figure 1).

varicose modes. The wall deformation is associated with a velocity field localised in thin layers close to the walls for short wavelengths.

\section{Optimisation results}

To investigate the sensitivity of the system to external perturbations, we proceed to compute initial conditions leading to large energy growth. We will see in the next sections that the mechanism of energy growth is related to the wall traveling waves. We can thus 


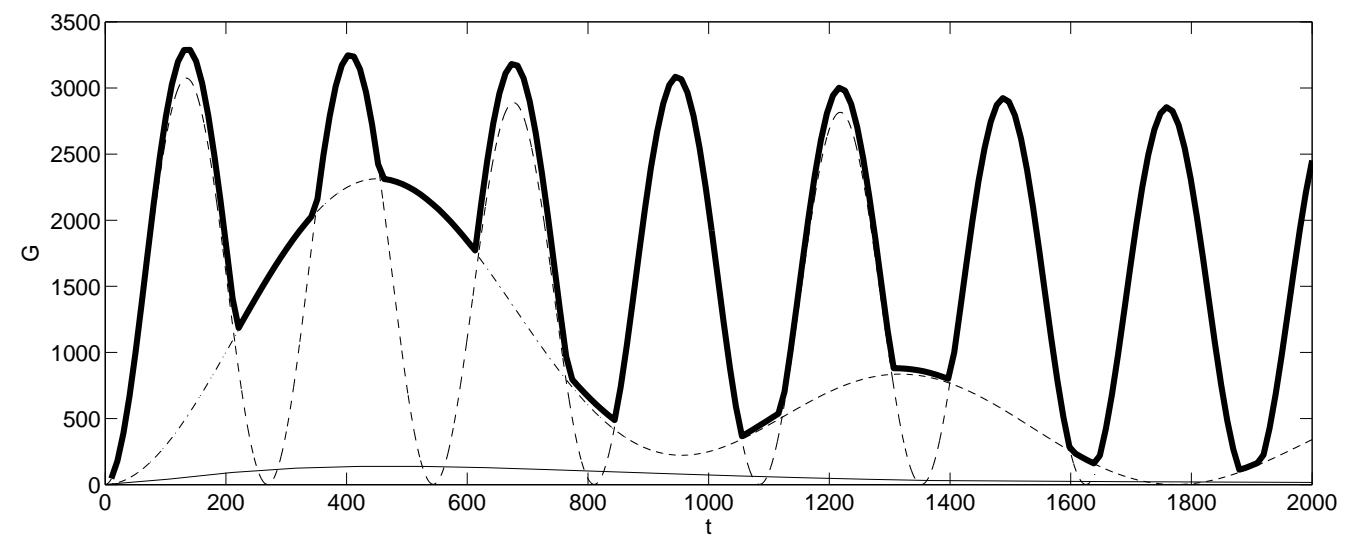

FiguRE 4. Thick line: envelope of maximum energy growth for $(\alpha, \beta)=(0,0.2), K=10^{4}$, $R e=5000, d=1$. We observe two oscillatory behaviours with apparent periods $T \approx 270$ and $T \approx 950$. The energy evolution of the initial conditions optimal for $t=400$ and $t=500$ are represented with dashed and dot-dashed lines. The optimal growth envelope for the rigid channel at $R e=5000$ is drawn with a thin solid line.

project the system onto its least stable eigenmodes. In the following we use 30 of these eigenmodes. The projected system is then described by

$$
\dot{\kappa}=\Lambda \kappa, \quad \mathcal{Q}=V^{H} Q V=F^{H} F,
$$

where $\kappa$ is the vector of expansion coefficients of the system's state on the retained eigenfunctions (flow velocity, pressure and wall displacement), $\Lambda$ is the diagonal matrix of the eigenvalues, $Q$ is the matrix of energy weights in physical space, $V$ the matrix whose columns are the eigenfunctions, $\mathcal{Q}$ is the energy weight matrix in eigenmode space (matrix of angles), and upperscript $H$ denotes the conjugate-transpose operation. We have further factored $\mathcal{Q}$ with Cholesky factor $F$. Using this formulation, we can compute for all times the maximum energy growth from an arbitrary initial condition $\kappa_{0}$ as

$$
G(t)=\max _{\kappa_{0}} \frac{\|\kappa(t)\|_{\mathcal{Q}}}{\left\|\kappa_{0}\right\|_{\mathcal{Q}}}=\max _{\kappa_{0}} \frac{\left\|e^{\Lambda t} \kappa_{0}\right\|_{\mathcal{Q}}}{\left\|\kappa_{0}\right\|_{\mathcal{Q}}} \triangleq\left\|e^{\Lambda t}\right\|_{\mathcal{Q}}=\|\underbrace{F^{-1} e^{\Lambda t} F}_{\mathcal{H}}\|_{2},
$$

where we have used the definition of the operator norm $\|\cdot\|_{\mathcal{Q}}$, and have expressed this $\mathcal{Q}$-norm in terms of the 2 -norm using $F$. In this expression, the operator 2-norm can be computed as the largest singular value of $\mathcal{H}$. The worst case initial condition, that is the initial condition that maximises this growth, is associated to the first right singular vector of $\mathcal{H}$, and the response is associated to its first left singular vector; see Schmid \& Henningson (2001) for further details.

A representative example of the growth envelope is shown in figure 4. We distinguish from the energy growth envelope the signature of two oscillatory mechanisms with different periods in time. The mechanism leading to the first peak arising after $t=0$ has sinuous symmetry, with an apparent period of about 270 time units. The second mechanism has varicose symmetry, with apparent period of about 950 time units. The energy evolution of initial conditions which are optimal for target times equal to 400 and 500 are shown as dashed and dash-dotted lines. From the matching between the energy evolutions and the envelope, we can infer that the energy growth envelope is mostly due to these two particular initial conditions.

The flow fields produced by these two initial conditions are displayed in figure 5 at different instants of time. We proceed here to the qualitative description of the phe- 

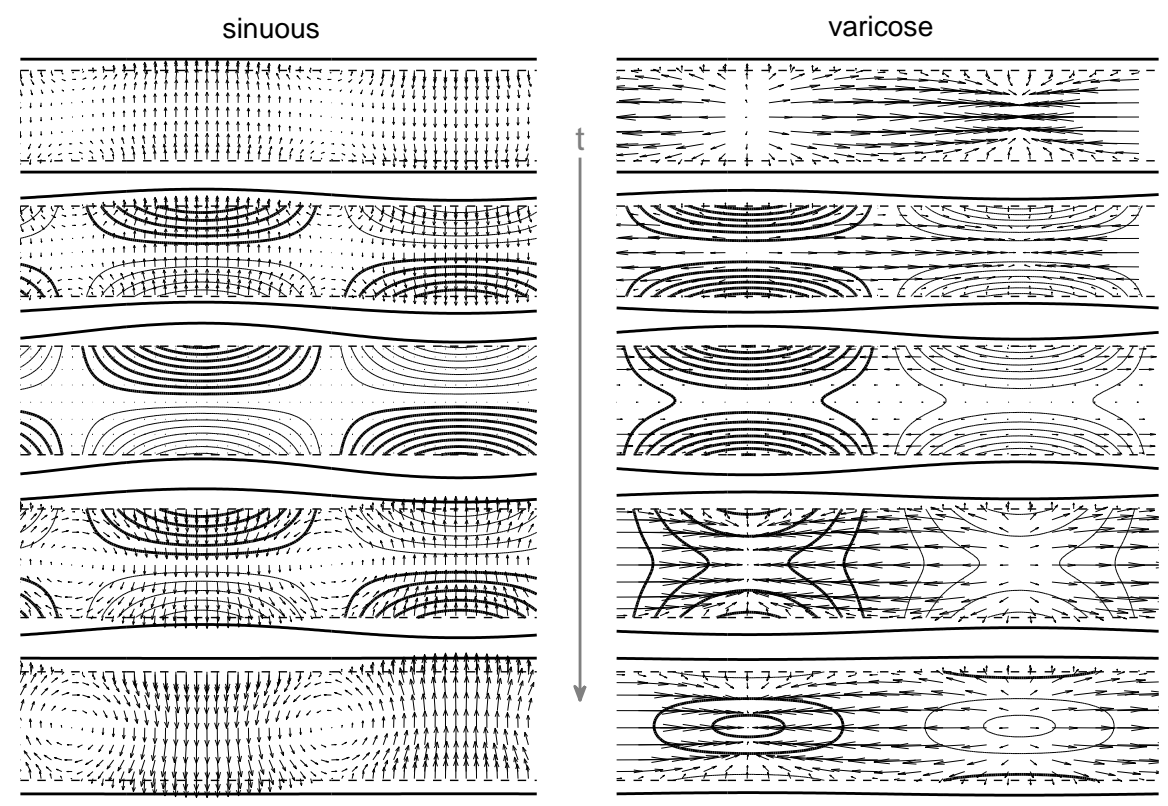

FIGURE 5. Evolution in the channel cross-section $(y, z)$ of the sinuous (left) and varicose (right) initial conditions shown in figure 4 . Snapshots at five linearly spaced instants of time (top row corresponds to $t=0$ ) within the apparent oscillation period for each of these initial perturbations, $v$ and $w$ velocity components are represented as vector fields, and the streamwise velocity $u$ is drawn with iso-contours: thick lines for positive velocity, and thin lines for negative (same parameters as in figure 4). Animations of the time evolution of the optimal perturbations are shown in movie 1, available with the online version of this paper.

nomenon; analysis and quantification will be performed in the next section. Note that the wall motion is enforced in the linear stability framework using inhomogeneous boundary conditions at $y= \pm 1$. We have nevertheless represented the wall deformation $\eta$ with thick solid lines for clarity (with arbitrary amplitude).

Initially $(t=0)$ the walls are straight and there is no streamwise velocity. The inplane velocity profile then generates wall deformations with sinuous symmetry for the short period oscillation and with varicose symmetry for the slower process. While the walls are deformed, a streamwise disturbance velocity component is generated by vertical advection of the base flow. At peak energy time (third row of plots in the figure), the disturbance flow is mostly composed by the streamwise component of the velocity. The motion is then reversed, as an effect of wall elasticity, until a situation close to the initial condition is reached, but with the disturbance velocity components of opposite sign. At this stage, the walls are straight again. For another apparent period of the energy curve, we observe the same process reversed (not shown). These oscillations have the structure of a standing wave, there is no propagation of the wall deformation in the horizontal direction $( \pm z)$. When describing the energy envelopes, we referred to the apparent period of the oscillatory mechanisms. It is now clear that these time intervals were in fact half of a complete period of oscillations (and figure 5 displays snapshot within half a period only). We further observe in figure 4 that the sinuous oscillation (of shorter period) is mildly damped, whereas the varicose oscillation is damped rapidly.

Similar oscillating curves of the gain have been obtained, for example, by de Luca \& Caramiello (2001) for the case of sinuous transient disturbances in plane liquid jets falling 


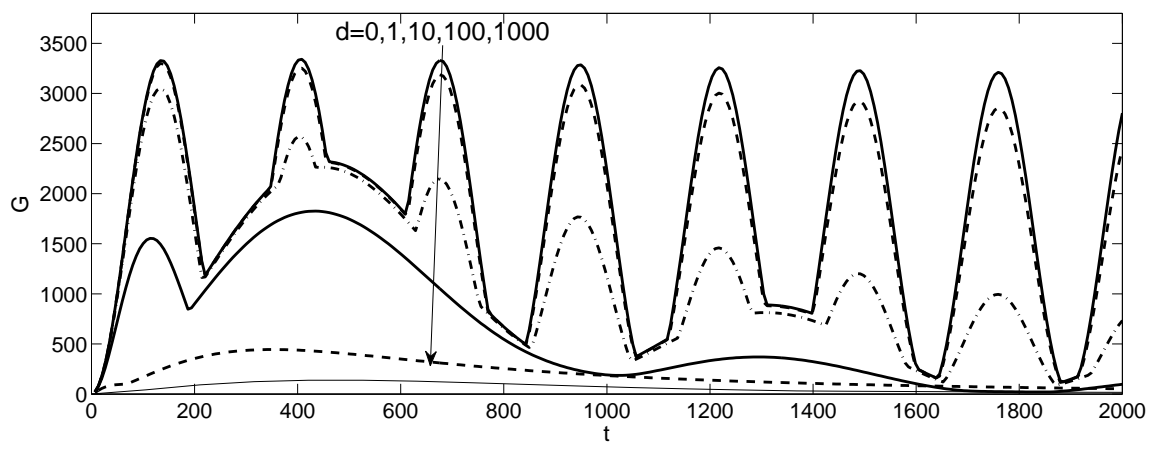

FiguRE 6. Effect of wall damping on energy growth. Parameters as by figure 4, with $d=0,1,10,100,1000$. The optimal growth envelope for the rigid channel is drawn with a thin solid line.

under gravity. In their case surface tension plays the role that the compliant coating plays here, inducing a mild algebraic growth. In the present case, the maximum energy gain is of order 1000 and decreases with the wall damping coefficient in the manner displayed in figure 6 . Up to $d=10$ the first peaks of the growth curves are almost unaffected; the oscillations which follow are more damped the larger $d$ is. For $d=100$, the varicose mechanism yields the largest transient amplification, and sinuous peaks have disappeared from the growth curves (aside from the first peak close to $t=100$ ).

\subsection{Mechanisms for growth}

Inspection of the velocity fields of figure 5 suggests simple mechanisms for the sinuous and varicose oscillations. The discussion is now based on figure 7 , where we have represented three possible oscillation scenarii: motion up and down of the entire channel and finite wavelength sinuous/varicose deformations. Considering these motions as perturbations to a static Poiseuille profile, we will quantify their energy evolution.

Let us consider first the up and down motion of the two walls (figure 7 left). A Poiseuille flow with spring-mounted walls that are slightly displaced from rest would sustain oscillations of frequency $\omega$, with $\omega^{2}=K / \operatorname{Re}^{2}\left(m+m_{a}\right)$, due to the springs, where the mass is that of the wall plus the added mass $m_{a}$ of the fluid. Denoting the wall displacement $\eta=\varepsilon \cos (\omega t)$, the parabolic velocity profile moving up and down is $\tilde{u}=1-(y+\eta)^{2}$. At first order in the small displacement $\eta$ the streamwise velocity is $1-y^{2}-2 \eta y$, so that the resulting time varying perturbation to the original (static) Poiseuille profile is $u(y)=-2 \eta y$. The associated vertical velocity $v$ will be constant in $y$ at each time instant, and will be equal to the displacement velocity of the wall $v=\eta_{t}$ in the whole channel. In the present context, we have $w=0$.

We can now quantify the energy evolution of this motion; the result is given below in (4.4). The total energy is

$$
E(t)=\frac{1}{2} \int_{y}\left[\tilde{u}^{2}+v^{2}\right] \mathrm{d} y+\left(m \eta_{t}^{2}+K \frac{\eta^{2}}{R e^{2}}\right),
$$

where we have accounted for the kinetic and potential energy of both walls. The streamwise contribution is:

$$
\int_{y} \tilde{u}^{2} \mathrm{~d} y=\int_{-1+\eta_{b o t}}^{1+\eta_{t o p}}\left[1-(y+\eta)^{2}\right]^{2} d y=\frac{16}{15}
$$



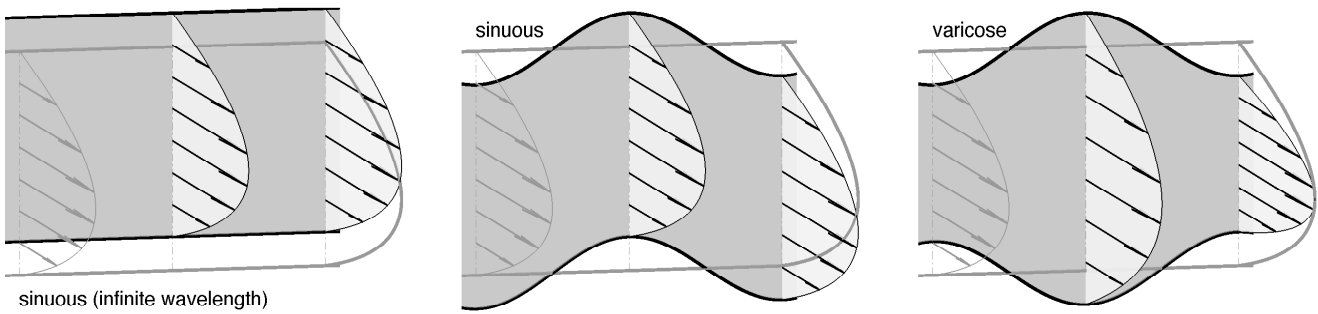

FIGURE 7. Sketch of the flow deformation induced by a spanwise standing wave. The Poiseuille base flow is displaced or stretched according to the channel deformation. Animation of these flow deformations are shown in movie 2, available with the online version of this paper.

Using $v=\eta_{t}=-\varepsilon \omega \sin (\omega t)$ we have:

$$
2 E(t)=\frac{16}{15}+2 \varepsilon^{2}\left[\omega^{2}(m+1) \sin ^{2}(\omega t)+\frac{K}{R e^{2}} \cos ^{2}(\omega t)\right] ;
$$

this total energy must be conserved in time, thus the coefficients of the sine and cosine terms should be equal, leading to:

$$
\omega^{2}=\frac{K}{R e^{2}(m+1)} .
$$

Comparing this expression with (3.2) at $\beta=0$, we obtain a confirmation of the unit added mass for long-wave sinuous modes. Turning now to the perturbation energy with respect to the static Poiseuille profile, we have:

$$
\int_{y} u^{2} \mathrm{~d} y=\varepsilon^{2} \cos ^{2}(\omega t) \int_{y}(2 y)^{2} \mathrm{~d} y=\frac{8}{3} \varepsilon^{2} \cos ^{2}(\omega t)
$$

The energy evolution of the perturbation is thus:

$$
2 E / \varepsilon^{2}=2 \underbrace{\left(\frac{4}{3}+\frac{K}{R e^{2}}\right)}_{A} \cos ^{2}(\omega t)+2 \underbrace{\left(\omega^{2}(m+1)\right)}_{B} \sin ^{2}(\omega t),
$$

which is the radius of an ellipse at an angle $\omega$ from its principal axis. Expressing $\omega$ as in (4.3), we obtain the energy growth along one fourth of the rotation period around the ellipse as:

$$
G_{\beta=0}^{s}=\frac{A}{B}=1+\frac{4}{3} \frac{R e^{2}}{K} .
$$

This oscillation mechanism conserves the system's total energy, but this particular perturbation to the static Poiseuille motion experiences an oscillatory energy evolution of potentially large amplitude when the walls are very flexible (low $K$ ). For very large stiffness, the maximum growth tends to unity, i.e. energy conservation of the perturbation. Following the same line of thought while considering a finite wavelength, we obtain the final relation

$$
G^{s}=1+\frac{4}{3} \frac{R e^{2}}{B \beta^{4}+T \beta^{2}+K} .
$$

For the varicose case, by analogy with the sinuous case, the expression

$$
G^{v}=1+\frac{14}{15} \frac{R e^{2}}{B \beta^{4}+T \beta^{2}+K}
$$

can be selected which shows a good fit to the numerical results. The varicose case is in 
fact more complex, mainly due to the flow from constricted regions to expanded regions; it is discussed in more details in $\S 5$ below.

In the numerical computations of the maximum transient growth we decouple the sinuous and varicose mechanisms ( $c f$. Trefethen (2000) $\$ 11$ for a description of how to enforce numerically one or the other symmetry). On figure 8 the maximum growth for $\alpha=0$ is shown for the sinuous parity. The energy growth of the rigid channel is also represented, depicted by the outer envelope of the shaded region. We can see that the model agrees well with the computed results, and that when the described mechanism leads to a lesser growth than that of the rigid system, the flexible channel gives the same result as the rigid one.

The equivalent result for the varicose symmetry is represented in figure 8c,d. For this case, the growth is compared to that of the rigid channel with imposed varicose parity ( $u$ is symmetric, $v$ is antisymmetric about the channel centreline). Similarly to the sinuous case, the oscillatory model agrees well with the computed results, and the flexible channel tends to behave as the rigid one whenever the rigid channel amplification exceeds that arising from the elasticity of the walls. Note that the transient growth in the channel with varicose symmetry, corresponds to the second singular value of the propagator $\mathcal{H}$ in the case of no parity enforced a-priori.

\section{The role played by wall modes in the optimal growth}

We introduced this paper by justifying the need for a non-modal analysis. In fact we will see in this section that some eigenmodes, namely those eigenmodes which correspond to wall traveling waves, play a central role in the standing wave mechanism. Surprisingly, the role of a pair of wall eigenmodes will be fully appreciable only when considering eigenmodes of the inviscid system.

First, we rewrite the disturbance equations in the inviscid limit, see (1.2), with boundary conditions $v=\eta_{t}$ at the compliant walls and slip conditions on the walls for $u$ and $w$. Here we must apply slip conditions at the walls since we consider the inviscid equations. The evolution of the $v$ and $w$ perturbation velocity components is that of a channel without base flow, subject to incompressibility and no-penetration at the walls. The streamwise velocity does not influence the behaviour of $v$ and $w$, but since $u_{t}=-U_{y} v$, the evolution of $u$ stems from $v$.

The flexible channel can sustain four propagative modes, arising from the free-wall case but with an added mass effect: two modes propagating in one direction, one sinuous and one varicose, and by symmetry two modes propagating in the opposite direction. We now consider two modes of one parity, propagating along $\pm z$. Owing to the symmetry $(w \rightarrow-w, z \rightarrow-z)$ in (1.2), the two waves propagating in opposite directions will generate precisely the same streamwise velocity $u$ due to the advection process discussed in the previous section. As they travel in the $\pm z$ direction, the associated $u$ will propagate as well. Combining the two travelling waves into a standing wave, we obtain a mechanism for cancellation/superposition of the streamwise velocity: in the in-phase/out-of-phase process of the two propagating modes, the associated $u$ will be added, then cancelled, periodically, leading to the observed oscillatory growth.

We now consider the amplitude of the $u$ velocity generated by each traveling wave individually, basing the discussion on (1.2). For a single eigenmode of frequency $\omega$ we have:

$$
i \omega \hat{u}+U_{y} \hat{v}=0 \quad \Rightarrow \quad\|\hat{u}\|=\frac{T^{2}}{4 \pi^{2}} \int_{y}\left|U_{y} \hat{v}\right|^{2} \mathrm{~d} y,
$$



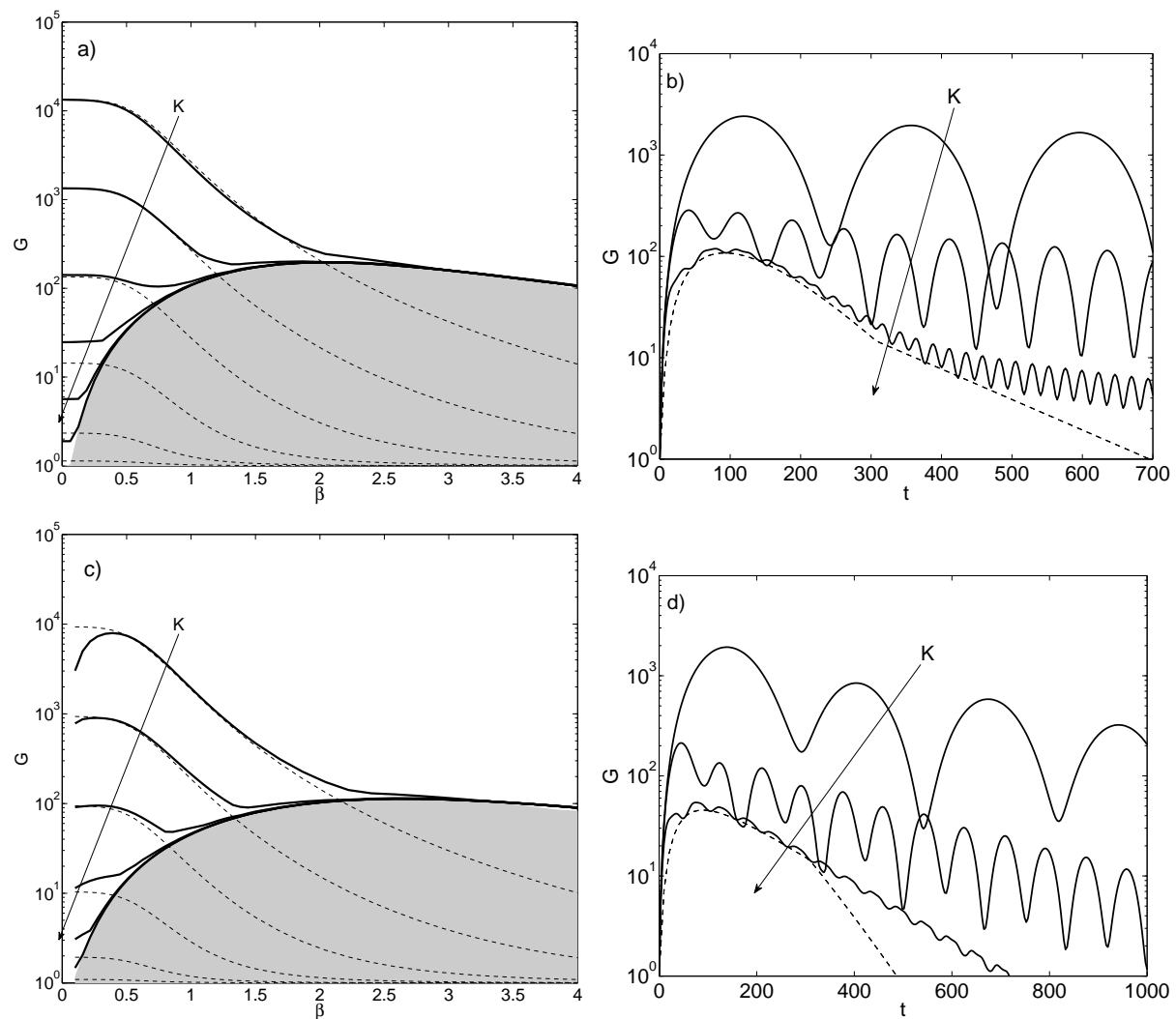

FIgURE 8. Maximum energy growth in the flexible channel for a) the sinuous and c) the varicose mechanisms while varying the spanwise wavenumber $\beta$ (thick solid) and for $\alpha=0$, $K=10^{2}, 10^{3}, \ldots, 10^{7}$. The results are compared to the oscillatory models (4.5) and (4.6) (thin dashed), and to the maximum growth of the rigid channel case (shaded area). In b) and d) the energy envelopes for sinuous and varicose modes are represented at $\beta=1, d=0, R e=1000$ for $K=10^{2}, 10^{3}$ and $10^{4}$, and compared to the optimal envelope for the rigid channel (dashed).

where $\|\hat{u}\|$ is the norm of the disturbance velocity, and $T=2 \pi / \omega$ is the period of the oscillation. In the case of cancellation/superposition effect of two counter-propagating traveling modes, the growth amplitude in $\|u\|$ will scale as $T^{2}$, that is, the slower the oscillation the larger the growth. Indeed, for an eigenmode with long time period, $v$ will advect the base flow velocity for a long time, generating strong perturbations, before reversing its advection direction. The growth in $u$ will depend also on the eigenmode shape $\hat{v}$, whether its support is located where large shearing $\left|U_{y}\right|$ is present. Note that to derive an estimate of the total energy oscillation in this standing wave process, we should also account for the kinetic and potential energies of the wall.

To evaluate the role played by each eigenmode in the energy growth, we perform the optimisation by increasing successively the number of selected eigenmodes, from 1 to 20; starting with the least stable ones. The results are shown in figure 9. For the sinuous case at long wavelength, two modes give the full growth. The flow evolution is that of a standing wave: the two wall modes have equal amplitude. For shorter wavelength more eigenmodes must be included. For the varicose symmetry, even at long wavelength the two wall modes cannot sustain the full growth, and two additional eigenmodes must be included. It is interesting to note that one of these modes has zero wall deformation (not 

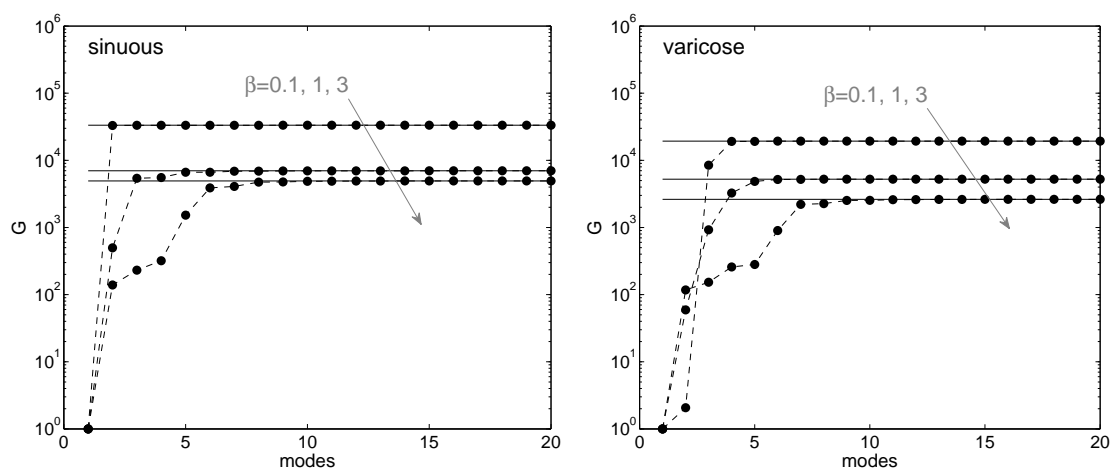

Figure 9. Optimal growth obtained by projection on an increasing number of eigenmodes for $K=10^{3}, R e=5000, d=0$.

shown here). We can also confirm from this figure that computing the optimal growth by projecting onto the 30 least stable eigenmodes is enough to obtain converged results.

Incidentally, we observe that we have been unable to obtain convergence towards the optimal solution when increasing too much the number of modes used in the projection. For example, by using the whole spectrum of eigenmodes the optimal initial condition suffers from severe dispersion errors, with numerical point-by-point oscillations. Attempts to regularise the objective function by introducing high-order derivatives of the velocity components did not yield the hoped-for smoothing of the numerical oscillations. Presumably the problem is due to the way the wall deformation is introduced in the system, by enforcing implicitly the inhomogeneous boundary conditions, but we have no definite evidence of this. We further note that the non-convergence of the disturbance energy with the increase of the number of modes seems to be a common problem in cases in which interfaces exist (Malik \& Hooper 2007); it is usually ascribed to the presence of spurious modes and/or to the nature of the functional for which optimisation is sought.

Figure 9 shows that it is only for the sinuous symmetry, at long wavelength, that two wall modes can form a standing wave strong enough to yield optimal growth. Since the mechanisms highlighted in $\S 4.1$ are in essence inviscid, one might suspect that it is rather the eigenmodes of the inviscid system which cause large energy gains. We have thus computed the energy growth from a standing wave built from two oppositely propagating wall modes, based on the eigenmodes of the viscous system, and compared it with that produced by the corresponding two eigenmodes of the inviscid problem. The energy evolution is oscillatory, the largest growth being the ratio of the maximum over the minimum energy in the course of these oscillations. The results are shown in figure 10. The energy growth curves obtained from the viscous eigenmodes (dashed curves) are consistent with results displayed in the previous figure: two modes underestimate the full growth for moderate $\beta$ except for the long spanwise-wavelength sinuous case. On the other hand, inviscid eigenmodes follow the trend of the oscillatory growth, except for the varicose case at long wavelengths. When the spanwise wavelength is long, the varicose modes generate a large momentum flux from constricted to expanded sections, as opposed to the sinuous mode for which at long wavelength flow particles just move up and down following the wall. This effect has two consequences, the first one being a large added mass effect in the sinuous case; the oscillation/propagation is slow for the long varicose waves, and this large flux induces strong viscous losses. Based on equation (5.1), slow oscillations should induce large energy growth; this is observed in figure 10 when viscosity is neglected. 

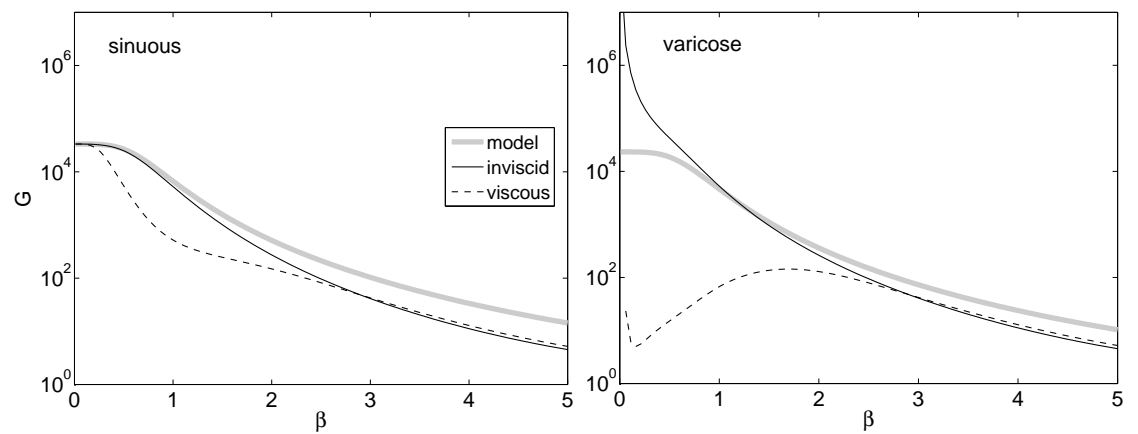

FIGURE 10. Energy growth for a standing wave composed of two oppositely propagating wall modes. Results are obtained from the eigenmodes of the inviscid case (dark solid) and viscous case (dark dashed), and are compared to the growth from the oscillatory models (4.5) and (4.6) (light grey) already employed for the comparisons presented in figure 8 . The parameters are $K=10^{3}, R e=5000, d=0$.

\section{Conclusions}

We have aimed at answering the question: 'Are there perturbations that can grow in a compliant channel?' and have indeed found three mechanisms. One is the classical mechanism of the rigid wall case, consisting in the formation of streaks from initial streamwise vortices. In addition, there are two spanwise standing wave oscillation processes, one sinuous (the walls are deformed antisymmetrically with respect to the channel centreline) and one varicose (walls symmetrical). These three processes concern primarily disturbances which do not vary in the streamwise direction $(\alpha=0)$. It is well known that for $\alpha \neq 0$, the system is unstable to flow-induced surface instabilities when the wall stiffness is low, or to Tollmien-Schlichting waves when the Reynolds number is large, but we have chosen here to focus on different mechanisms which might dominate the initial dynamics under specific receptivity conditions. The effects examined are based on the interaction of wall modes, that is the flow/walls structures originating from travelling waves of the flexible plates.

Our main results are summarised by figures 7 and 10. The computed optimal energy growth can be explained from simple system deformations where the Poiseuille flow is displaced in the vertical direction in a sinuous shape, or stretched/contracted in a varicose manner. A quantification of the energy evolution for this simple model shows good agreement with the computed data, thus confirming our description of the mechanisms at play. The period of the standing-wave oscillations can be easily predicted from the dispersion relation of free-wall waves, modified to account for an added mass effect due to the presence of the fluid (cf. equation 3.4).

These mechanisms are most energetic when the walls are very elastic and the forcing disturbance spectrum includes long spanwise wavelengths. Under these conditions the compliant channel is sensitive to external perturbations. Note that almost all of the energy growth is carried by the streamwise velocity perturbation, as a result of the up/down advection of the base flow profile. In the case of anisotropic wall properties, where for instance waves which are initially amplified by the exponential mechanism at $\alpha \neq 0$ are eventually damped by an increasing rigidity in the longitudinal direction, the standing wave shown in figure 4 and 5 should dominate the overall system behaviour.

It is now interesting to discuss whether these mechanisms are likely to trigger transition to turbulence. The oscillations at infinite wavelength consist of up and down motion of the entire channel plus the walls. The total energy is conserved, but the perturbation 
with respect to the static reference parabolic profile follows a large amplitude oscillatory evolution. Probably here, as it is also the case for long sinuous waves, the energy growth with respect to the static profile does not imply strong flow distortions (hence no strong secondary instabilities should be expected). For the varicose symmetry on the other hand, the Poiseuille profile is not simply advected up and down, but it is stretched and contracted, in a motion associated with particles flowing from constricted to expanded regions. This distortion is more complex and it is likely that the velocity gradients thus generated be of relevance to transition to turbulence.

The type of analysis presented in this paper, where optimisation is used to put in evidence mechanisms of energy growth, is commonly referred to as non-modal. This term might however be misleading here since two spanwise counter-propagating eigenmodes, associated with the traveling waves of the flexible plates, are sufficient to generate a standing wave able to extract energy efficiently from the base flow. In this sense, this $b i$ modal mechanism is different from that discussed for example by Cossu \& Chomaz (1997), Schmid \& Henningson (2002) and Ehrenstein \& Gallaire (2005), whereby eigenmodes can collectively generate transient growth by forming localised wave packets.

Support from the EU in the form of a Marie Curie EST grant (project FLUBIO, MESTCT-2005-020228) is gratefully acknowledged. AB wants also to acknowledge interesting discussions with Luigi de Luca on the topic of this paper.

\section{REFERENCES}

Benjamin, T. B. 1960 Effects of a flexible boundary on hydrodynamic stability. J. Fluid Mech. 9, 513-532.

Carpenter, P. W., Davies, C. \& Lucey, A. D. 2000 Hydrodynamics and compliant walls: does the dolphin have a secret? Curr. Sci. 79, 758-765.

Carpenter, P. W. \& Garrad, A. D. 1985 The hydrodynamic stability of flow over Kramertype compliant surfaces. Part 1. Tollmien-Schlichting instabilities. J. Fluid Mech. 155, 465-510.

Carpenter, P. W. \& Garrad, A. D. 1986 The hydrodynamic stability of flow over Kramertype compliant surfaces. Part 2. Flow-induced surface instabilities. J. Fluid Mech. 170, $199-232$.

Carpenter, P. W. \& Pedley, T. J., ed. 2003 Flow Past Highly Compliant Boundaries and in Collapsible Tubes, Fluid Mechanics and Its Applications, vol. 72, IUTAM Symposium. Springer.

Cossu, C. \& Chomaz, J.-M. 1997 Global measures of local convective instabilities. Phys. Rev. Lett. 78, 4387-4390.

Davies, C. \& Carpenter, P. W. 1997 Instabilities in a plane channel flow between compliant walls. J. Fluid Mech. 352, 205-243.

Ehrenstein, U. \& Gallaire, F. 2005 On two-dimensional temporal modes in spatially evolving open flows: the flat plate boundary layer. J. Fluid Mech. 536, 209-218.

Fish, F. E. \& LAuder, G. V. 2006 Passive and active flow control by swimming fishes and mammals. Ann. Rev. Fluid Mech. 38, 193-224.

Gray, J. 1936 Studies in animal locomotion. VI. the propulsive power of dolphins. J. Exp. Biol. 13, 192-199.

Guaus, A. \& Bottaro, A. 2007 Instabilities of the flow in a curved channel with compliant walls. Proc. R. Soc. A 463, 2201-2222.

Gad-El HaK, M. 2000 Flow control. Passive, active, and reactive flow management. Cambridge University Press.

Kramer, M. O. 1957 Boundary-layer stabilization by distributed damping. J. Aerosp. Sci. 24, 459-460.

Kramer, M. O. 1960 Boundary-layer stabilization by distributed damping. J. Am. Soc. Navig. Eng. 72, 25-33. 
LANDAHL, M. T. 1962 On the stability of a laminar incompressible boundary layer over a flexible surface. J. Fluid Mech. 13, 609-632.

DE LuCA, L \& CARAmiello, C. 2001 Temporal growth of perturbations energy in plane heterogeneous jets. AIAA Paper (2704).

Malik, S. V. \& Hooper, A. P. 2007 Three-dimensional disturbances in channel flows. Phys. Fluids (052102).

Schmid, P. \& Henningson, D. 2002 On the stability of a falling liquid curtain. J. Fluid Mech. 463, $163-171$.

Schmid, P. J. \& Henningson, D. S. 2001 Stability and transition in shear flows. Springer, New York.

Trefethen, L. 2000 Spectral Methods in Matlab. SIAM.

Weideman, J. A. \& ReDdy, S. C. 2000 A matlab differentiation matrix suite. ACM Trans. Math. Softw. 26 (4), 465-519.

Wiplier, O. \& Ehrenstein, U. 2001 On the absolute instability in a boundary-layer flow with compliant coating. Eur. J. Mech. B - Fluids 20, 127-144. 